\title{
Experimental and numerical determination of Darcy's law for yield stress fluids in porous media
}

\author{
D. Bauer, ${ }^{1}$ L. Talon, ${ }^{2,}{ }^{*}$ Y. Peysson, ${ }^{1}$ H. B. Ly, ${ }^{1}$ G. Batôt, ${ }^{1}$ T. Chevalier, ${ }^{1}$ and M. Fleury ${ }^{1}$ \\ ${ }^{1}$ IFP Energies nouvelles, 92852 Rueil-Malmaison, France \\ ${ }^{2}$ Laboratoire FAST, Univ. Paris-Sud, CNRS, Université Paris-Saclay, F-91405, Orsay, France
}

(Received 22 June 2018; published 5 June 2019)

\begin{abstract}
In this work, we studied experimentally and numerically the pressure-flow rate relationship for yield stress fluids in porous media. We developed and validated 3D numerical simulations of the velocity field via a lattice Boltzmann method based on the TRT scheme, and a specific experimental setup allowing yield stress fluids to flow in a closed-loop system to obtain stable rheological properties over a wide range of flow rates (4 decades). The porous medium studied experimentally is a sandstone. The flow properties were also simulated on a regular sphere packing and a random sphere packing as well as on a 3D geometry of a sandstone obtained by x-ray tomography. These different geometries allow highlighting the role of the heterogeneity of the pore structure on the flow properties. All results are expressed as the flow rate $\tilde{Q}$ versus the difference of the pressure gradient to the critical pressure $\left(\Delta \tilde{P}-\Delta \tilde{P}_{c}\right) ; P_{c}$ defines the pressure below which there is no flow. We observed both numerically and experimentally three specific scaling regimes, already identified by Talon and Bauer [Eur. Phys. J. E 36, 139 (2013)] for a Bingham fluid in 2D porous media. We also evidenced the existence of two critical pressures: the "true" critical pressure $\tilde{P}_{c}$ defined as the pressure below which there is no flow and the "pseudo" critical pressure threshold $\tilde{P}_{c}^{\infty}$ determined by fitting the data in the high-flow-rate regime. We show that the "true" critical pressure is always lower than the "pseudo" one in heterogeneous porous media and can be equal only in the case of regular porous structures. We explain these observations using an energy minimization principle.
\end{abstract}

DOI: 10.1103/PhysRevFluids.4.063301

\section{INTRODUCTION}

Among the very large variety of non-Newtonian fluids, many of them display a yield-stress behavior. If the applied stress is below the plastic threshold, then the response might be a deformation but not a rate of deformation (shear rate). Once the applied stress is sufficiently high, the material is sheared and thus flowing. The yield-stress property is shared by different physical systems, such as emulsions, suspensions, polymer solutions, gels, foams, or granular media. Yield-stress fluids, also named viscoplastic fluids, are thus encountered in many applications, such as cosmetics or food industry, civil engineering, soil mechanics, etc. In the context of porous or fractured media, mud, cement, or heavy oil are noticeable yield-stress materials. Important processes like water filtration in clay, well drilling, hydraulic fracturation, ground cementing, or enhanced oil recovery involve viscoplastic flow in porous media. It is then not surprising that the study of yield-stress fluid has been the subject of numerous studies for over half a decade [1-15]. As often in problems dealing with porous media, a major task is the derivation of a constitutive equation at

*talon@fast.u-psud.fr 
large scale which relates the applied pressure drop, $\Delta P$, to the average flow rate in the medium, $Q$, which generalizes Darcy's law to yield stress fluid.

A common constitutive law describing yield-stress fluids is the Herschel-Bulkley law, which states that the material responses like a nondeformable solid below the threshold $\left(\tau_{0}\right)$ and like a power-law fluid above,

$$
\begin{cases}\dot{\gamma}^{n}=\frac{1}{k}\left(\tau-\tau_{0}\right) & \text { if } \tau \geqslant \tau_{0} \\ \dot{\gamma}=0 & \text { otherwise, }\end{cases}
$$

$\tau$ is the shear stress, $\tau_{0}$ the critical yield stress, $\dot{\gamma}$ the shear rate, $k$ the consistency, and $n$ the flow index.

Using this rheological law, most of the constitutive equations based on theoretical, numerical, or experimental data have the same structure. They are referred to as limiting pressure gradient (LPG) [2] and given by

$$
Q^{n}=A\left(\Delta P-\Delta P_{c}\right),
$$

where $\Delta P_{c}$ is a critical threshold pressure below which there is no flow.

With regard to the highly nonlinear constitutive rheology, it is striking that such simple law allows the correct description of flow in heterogeneous porous media. Indeed, if one considers a single tube, which represents the simplest porous medium, then the variation of the flow rate as a function of the applied pressure drop is more complex as it involves, in contrast to Eq. (2), several power-law terms [see Eq. (C8)].

For instance, as reported by $[14,16]$, one important consequence of these extra-terms is that in the high-flow-rate limit, the LPG is valid but with a value of the critical pressure that does not correspond to the onset of flow. Following [14], we will refer to this value as the "pseudo" critical pressure to distinguish from the "true" critical pressure. In this paper, we will discuss the physical origin of these two thresholds.

To take into account the disorder of the porous medium, fiber bundle models with randomly distributed radii predict a more sophisticated expression due to variations in the number of flowing channels when the applied pressure is increased [4,6,12]. The apparent simplicity of the Darcy's law [Eq. (2)] has been thus the subject of controversies. For instance, in Chevalier et al. [11], using NMR measurements in monodisperse glass bead packing, the authors conclude to the "breaking" of the non-Newtonian character of the fluid in porous media.

Yet, in an almost simultaneous work, using simulations of Bingham fluid $(n=1)$ in stochastic porous media, Talon and Bauer [1] have demonstrated the existence of at least three scaling regimes:

$$
\Delta P=A_{i} Q^{\alpha_{i}}+B
$$

By increasing the flow rate, $\alpha_{i}$ equals successively to 1,2 , and 1 . The physical origin of these exponents have been investigated in Chevalier and Talon [17] and are attributed to the structural disorder of the medium. The statistical properties of the flow structure is indeed characterized by power-law distributions reminiscent to critical systems (e.g., percolation). A notable result of this work is the independence of these power exponents with the type of disorder. $B$ is equal to the true critical pressure required for the onset of the flow in one channel.

To our knowledge, the existence of such regimes has never been observed, neither experimentally nor numerically, in 3D natural porous media and with Herschel-Bulkley fluids which is the purpose of this paper. We have developed an experimental setup allowing the investigation of the flow regimes in sandstone. In parallel, we have also performed 3D LB simulations of HershelBulkley fluid in monodispersed sphere packing and in a 3D microstructure determined from $\mathrm{x}$-ray microtomography of a sandstone. The structure of the article is as follows. We first present the experimental setup and the different porous structures used for the experiments and numerical investigation. In the result section, we show the obtained flow-rate pressure relation and the critical pressure. Finally, we discuss the influence of the irregular structure on the critical pressure. In the 
Appendix, we present in detail the rheology measurement, the numerical method and its validation in $3 \mathrm{D}$ structures, and the analytical determination of the critical pressure.

\section{MATERIALS AND METHODS}

\section{A. The Stokes equations}

Let us first recall the governing equations defining the creeping flow for Herschel-Bulkley fluids:

$$
\nabla \cdot \boldsymbol{\Pi}-\nabla P=\mathbf{0},
$$

where $\Pi$ is the shear stress tensor and $P$ is the pressure. The shear stress tensor for a HerschelBulkley fluid follows:

$$
\boldsymbol{\Pi}_{i j}=2 \rho v_{\mathrm{eff}} \boldsymbol{\Delta}_{i j}
$$

where the effective viscosity $v_{\text {eff }}$ is given by

$$
v_{\mathrm{eff}}=\left(k \dot{\gamma}^{n-1}+\frac{\tau_{0}}{\dot{\gamma}}\right) .
$$

$\boldsymbol{\Delta}_{i j}$ is the deformation rate tensor expressed as

$$
\boldsymbol{\Delta}_{i j}=\frac{1}{2}\left(\frac{\partial \boldsymbol{u}_{i}}{\partial x_{j}}+\frac{\partial \boldsymbol{u}_{j}}{\partial x_{i}}\right)
$$

and, using Einstein's summation convention,

$$
\dot{\gamma}=\sqrt{2 \Delta_{i j} \Delta_{i j}}
$$

is the shear rate. $\boldsymbol{u}$ is the velocity field, $\tau_{0}$ the yield stress, $k$ the consistency index, $n$ the flow index, and $\rho$ the density.

\section{B. Nondimensional flow rate and pressure difference}

Hereinafter, the total flow rate and the pressure will be nondimensionalized using the rheological properties of the fluid and the porous medium geometry. The total flow rate is nondimensionalized as

$$
\tilde{Q}=\frac{Q}{S \lambda}\left(\frac{k}{\tau_{0}}\right)^{1 / n},
$$

where $S$ is the entry section area and $\lambda$ a characteristic length scale which corresponds either to the average sphere radius for FCC and irregular bead packing or the estimated average grain size for the sandstones $(\lambda=16.9 \mu \mathrm{m})$. Notably, this nondimensional flow rate is related to the standard definition of the Bingham number $\mathrm{Bi}=\left(\frac{S \lambda}{Q}\right)^{n}\left(\frac{\tau_{0}}{k}\right)=\tilde{Q}^{-1 / n}$.

Similarly, the pressure difference is nondimensionalized as

$$
\Delta \tilde{P}=\frac{\Delta P \lambda}{\tau_{0} L},
$$

where $L$ is the length of the sample in the flow direction.

\section{Experimental investigation}

\section{Rheology}

Carbopol gels, a polymer based on polyacrylic acid $(-\mathrm{CH} 2-\mathrm{CHCOO}-)_{n}$, is a typical yield stress fluid. As their rheological properties can easily be represented by a Herschel Bulkley model [18], Carbopol gels have attracted the focus of researchers over the past decade [19] and became a reference fluid in the literature [19,20]. The nature of its yielding behavior [19] comes from a 


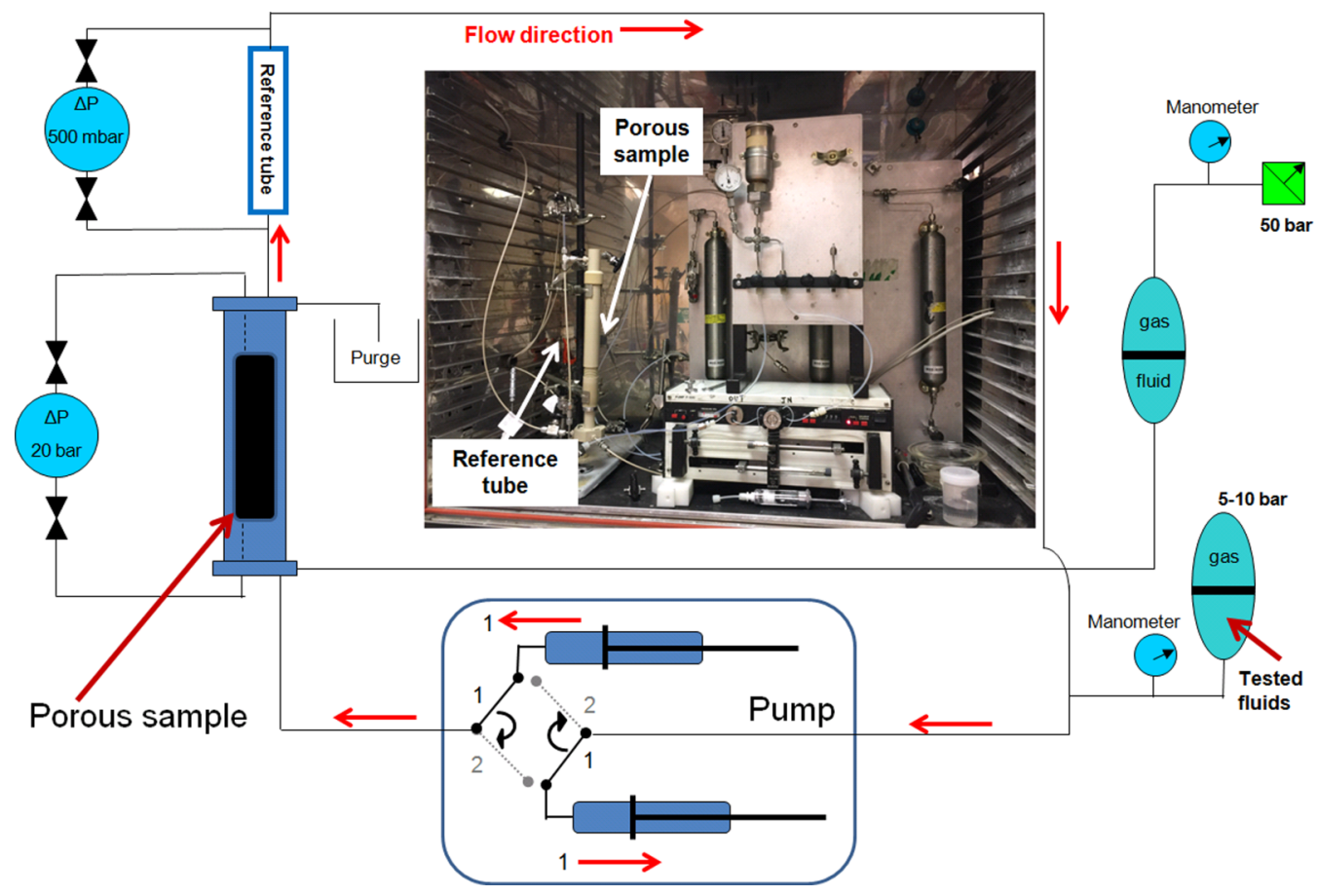

FIG. 1. Schematic diagram of the closed-loop setup used for Carbopol injection in the porous medium. The inset displays a photograph of the real system.

microstructure made of a high concentration of individual, elastic sponges (with a typical element size of $2-20 \mu \mathrm{m}$ ). The advantage of this gel is that it shows limited thixotropy and phase migration, making it a quite appropriate yield stress fluid for fundamental studies.

Carbopol rheology was determined for different concentrations $(0.1 \%, 0.25 \%, 0.5 \%$, and $1 \%$ in weight) using a MCR 300 rheometer. More details on the fluid preparation, measurements, and rheological properties are given in Appendix A.

\section{Experimental setup: The closed-loop system}

The experimental setup is mainly composed of a flooding cell in which a porous medium is inserted, a reference tube for monitoring the rheological properties of the flowing fluid, and a continuous flow pump made of two glass tubes in which two pistons are sliding in opposite directions (see Fig. 1). The tested fluid is flowing through the porous medium and the reference tube in a closed-loop circuit with an internal pressure set by a buffer in which about half the volume is occupied by nitrogen at the desired pressure (between 5 and 10 bars), and the other half is filled with the tested fluid. In this buffer, an elastomer membrane is present between the gas and the liquid to avoid gas dissolution into the flowing fluid. For the flooding cell, an elastomer membrane is also present around the sample with a confining pressure of 50 bar, much higher than the pore pressure (standard Hassler type cell). All these elements were put in an oven to stabilize the temperature at $30^{\circ} \mathrm{C}$. Temperature is measured with a thermometer placed close to the flooding cell. The variations were within $0.1^{\circ} \mathrm{C}$.

The porous media sample is a sandstone with a water permeability of $2050 \mathrm{mD}$ and a porosity of $\sim 20 \%$ (length $40.0 \mathrm{~mm}$, diameter $19.8 \mathrm{~mm}$ ). The reference tube has a length of $27.3 \mathrm{~cm}$ and an inner diameter of $2.0 \mathrm{~mm}$ determined by gravimetric method (the tube was weighted empty and water filled). 
The closed-loop circuit under moderate pressure was a key for successful experiments: First it allowed the pump to run at high flow rate (up to $500 \mathrm{ml} / \mathrm{h}$ ) without degassing problems of the fluid. Indeed, one of the cylinders of the pump must be filled while the other is pushing the fluid into the porous sample. Without the pressure imposed by the buffer, this would not have been possible due to the high effective viscosity of the investigated fluid. The glass tubes of the pump also allowed to visually control that no air was present. Second, a fluid with stabilized rheological properties could be analyzed. The rheological properties of the Carbopol mixture changed first during a transient regime flowing through the porous system and stabilized after some time. The pressure drop measurements were also carefully validated. Specific lines connected to the inlet and outlet faces of the porous medium were used independently of the injection tubings.

However, in theory, if the tubings connecting the pressure transducer to the inlet/outlet face are filled with the yield-stress fluid, then there is a possibility that the pressure drop is not measured correctly because the fluid at rest could act as a solid barrier not transmitting the pressure. To avoid this potential ambiguity, the lines and tubings connected to the pressure transducers were filled with a refined oil (dodecane). Doing so and without porous media, we verified that the rheological properties determined from the reference tube were identical to those determined from the rheometer.

\section{Experimental procedure}

The experimental procedure is as follows. First, the porous sample is saturated with a brine solution $(20 \mathrm{~g} / 1 \mathrm{NaCl})$ and its porosity measured by weight difference between the dry and saturated state. Then it is put in the flooding cell with a confining pressure. In parallel the Carbopol mixture is injected with a syringe in the buffer and the two cylinders of the pump are filled. Bypassing the flooding cell, a closed loop circulation is first realized to remove air bubbles trapped in the circuit. Then the flooding cell is connected to the system and the brine present in the porous medium replaced by the Carbopol mixture. Then, the reference tube is connected. The scanning of different flow rates is only started when the pressure drop at a moderate flow rate $(20-50 \mathrm{ml} / \mathrm{h})$ is stabilized; otherwise, the process is repeated. For a given fluid mixture, we first start at high flow rate $(500 \mathrm{ml} / \mathrm{h})$, then decrease it step by step to the lowest flow rate $(0.01 \mathrm{ml} / \mathrm{h})$. For each flow rate, the pressure drop is taken as an average of all stable values over a certain time period (about $1 \mathrm{~h}$ for low flow rate and $20 \mathrm{~min}$ for the rest). From one mixture to another, we checked after careful washing with brine that the permeability was unchanged, showing that no polymer was adsorbed in the porous medium or that no flow path was clogged by the polymer. Additionally, identical experiments were performed with glycerol as reference fluid for comparison.

\section{Numerical implementation}

\section{Lattice-Boltzmann scheme: Two relaxation time}

To solve the Stokes equation, we use a numerical approach based on a two relaxation time (TRT) scheme, which is well detailed in previous works of Talon and Bauer [1] or Ginzburg et al. [21-23]. Such works considered a two-dimensional (2D) Stokes flow of a Bingham fluid in porous media. In this work, we develop a 3D numerical code to solve Stokes flow of a Herschel-Bulkley fluid in porous medium. A brief description of the lattice-Boltzmann TRT scheme is presented in Appendix C 1 .

\section{Regularization of the effective viscosity}

From a numerical point of view, Eq. (6) is a source of instabilities for very low shear rate due to a diverging term $\left(\tau_{0} / \dot{\gamma}\right)$ in the effective viscosity expression. This is a well-known problem for yield stress fluids such as Bingham or Herschel Bulkley, that may be circumvented by using a regularized viscosity function. We use the one proposed by Papanastasiou et al. [24], which introduces an 

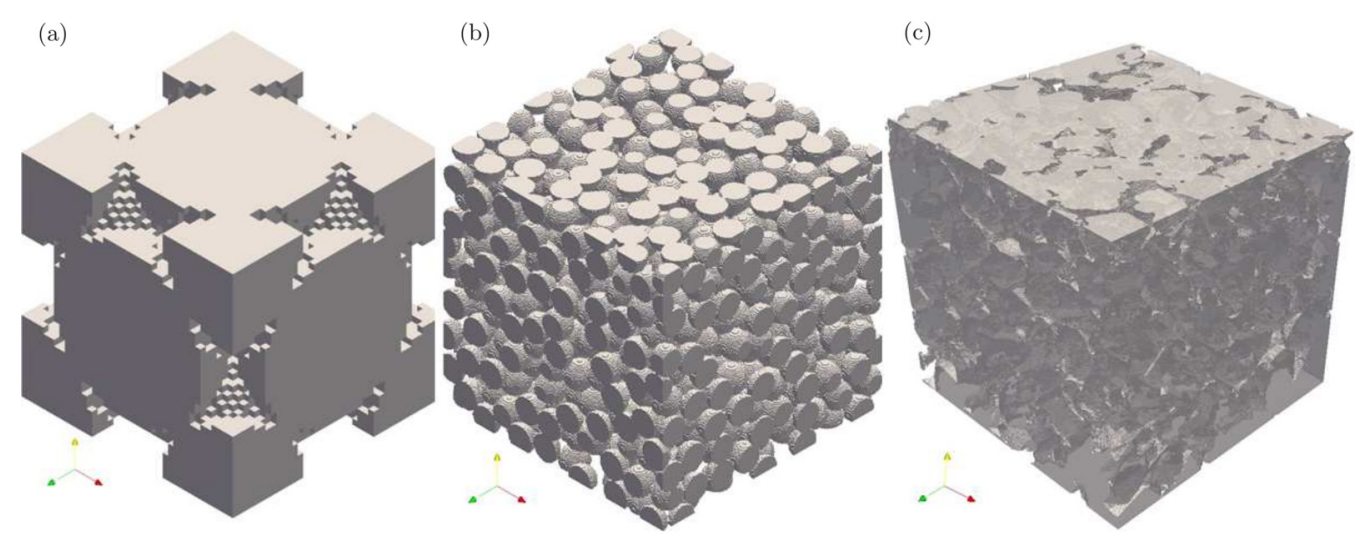

FIG. 2. Different porous media used in the simulation: (a) FCC packing of spheres; (b) random array of overlapping spheres; (c) 3D $\mu$-CT image of sandstone.

exponential function with a small parameter $\dot{\gamma}_{0}$ :

$$
v_{\mathrm{eff}}(\dot{\gamma})=k \dot{\gamma}^{n-1}+\frac{\tau_{0}}{\dot{\gamma}}\left(1-e^{-\frac{\dot{\gamma}}{\gamma_{0}}}\right)
$$

Strictly speaking, the fluid is thus never solid below the critical shear rate in numerical simulations but remains extremely viscous, and its viscosity is characterized by the parameter $\dot{\gamma}_{0}$. Physically, this parameter represents the typical shear rate below which the rheological law deviates from the original Herschel-Bulkley. Validations can be seen in Appendix C 3.

\section{Porous medium}

In this section, we present the different porous configurations used in our simulations: two computed idealized microstructures and a digital image of a real rock obtained by 3D microtomography. To mimic real porous media containing an interconnected solid phase and an interconnected porous phase, we first consider numerically generated sphere packings. We propose herein the use of two types of idealized porous media: a regular array of solid spheres positioned in a face-centered cubic (FCC) system [Fig. 2(a)] and a porous medium consisting of a random array of overlapping spheres [Fig. 2(b)]. Each cube has the dimension $L^{3}$. The radius of the sphere is $R=0.36 L$ for the FCC structure. For the random array of packing spheres 950 identical spheres of radius $R=0.055 L$ were used. The generation of such random porous media can be seen in Ref. [25]. Porosity of the porous medium is $\phi=21.8$ for the FCC and $\phi=42.9$ for the random packing array.

We finally consider the porous structure of a sandstone [Fig. 2(c)]. To this goal a 3D image of a small sample of sandstone was acquired by an x-ray microtomography system using a spatial resolution of $6 \mu \mathrm{m}$. Segmentation of the acquired gray level image was then performed by thresholding at the minimum between the two peaks of the gray level histogram resulting in a porosity of $14.5 \%$. The experimentally obtained permeability of this sandstone is $\sim 600 \mathrm{mD}$.

As LB methods use a regular, cubic grid, the voxel map obtained from the micro-CT image of the sandstone was directly used for LBM simulations. Thus, the resolution of the average channel restriction of the sandstone is fixed by the resolution of the micro-CT equipment. To have a comparable discretization error, synthetic microstructures in the simulations were discretized with a similar number of voxels for the average channel restriction. We used $40^{3}$ voxels for the FCC system, $256^{3}$ for the random packing, and $512^{3}$ for the sandstone.

Validation of the code as well as the influence of the grid resolution are given in Appendix C2. 


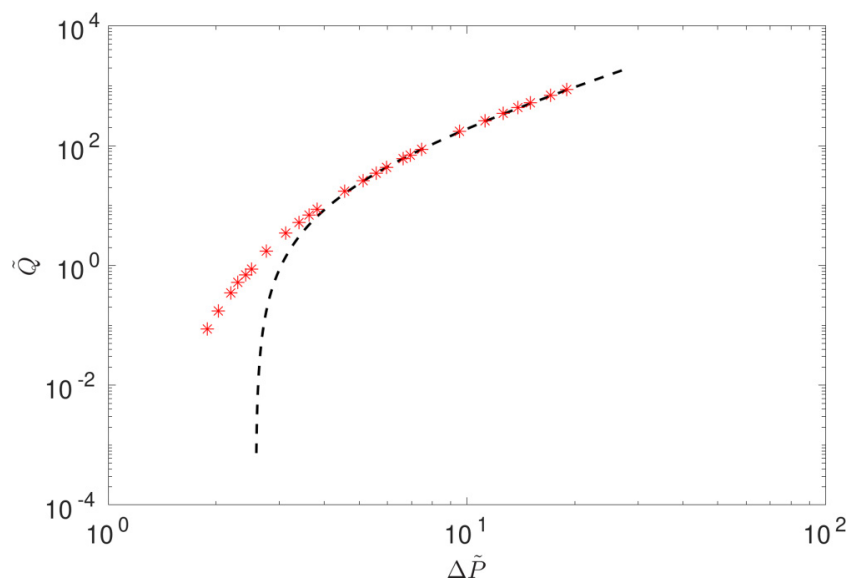

FIG. 3. Experimental flow rate of the sandstone as a function of the pressure drop. Stars represent experimental data, and dashed lines represent the fit with Eq. (12) considering the high flow rate.

\section{RESULTS}

\section{A. Experimental results}

We present the results of the $1 \%$ solution, as its yield stress is sufficiently significant to be measured. For lower concentrations, this behavior is less pronounced and its determination becomes therefore more imprecise with our equipment. Thus, we present only the results obtained with the $1 \%$ solution.

\section{Fluid evolution due to the porous structure}

Before analyzing the data, we first discuss an important aspect which is the evolution in time of the fluid while flowing through the porous medium. Indeed, when the flow rate $Q$ is imposed by the pump, the pressure drop $\Delta P$ might take a significant time to stabilize both in the porous medium and reference tube.

By continuously monitoring the pressure drop across the reference tube at imposed constant flow rate during 1 week, we can distinguish between a transient regime and a stationary regime. Only the data of the stationary regime is later on used for the determination of the generalized Darcy equation. In addition, rheological measurements were performed before and after the experiments for glycerol and Carbopol. For the yield stress fluid, there is an evolution of the critical shear stress $\tau_{0}$ and consistency $k$. The $n$ exponent varied only slightly (see Appendix B). However, no evolution of the glycerol has been observed. We believe that the evolution of the yield stress fluid is due to a slight degradation of the polymer gel structure caused by the flow in the porous sample and not to the hydraulic circuit before and after the porous medium in which various valves and connectors are present. Indeed, such evolution was not observed in the absence of porous medium with the same circuit. In addition, since the permeability of the porous medium did not change during the entire experimental program, adsorption effects cannot explain such evolution. Most likely, the pore throats might provoke some breakage of the polymer sponges or interconnection causing a decrease of the yield stress properties. A detailed explanation is outside the scope of this article. The important aspect is that the rheological properties are stable in the stationary regime in all investigated flow rates.

\section{Flow-rate-pressure relationship}

Figure 3 shows the nondimensional flow rate $\tilde{Q}$ as a function of the nondimensional pressure drop $\Delta \tilde{P}$. To determine the pseudocritical pressure $\Delta \tilde{P}_{c}^{\infty}$ the experimental data were fitted using 


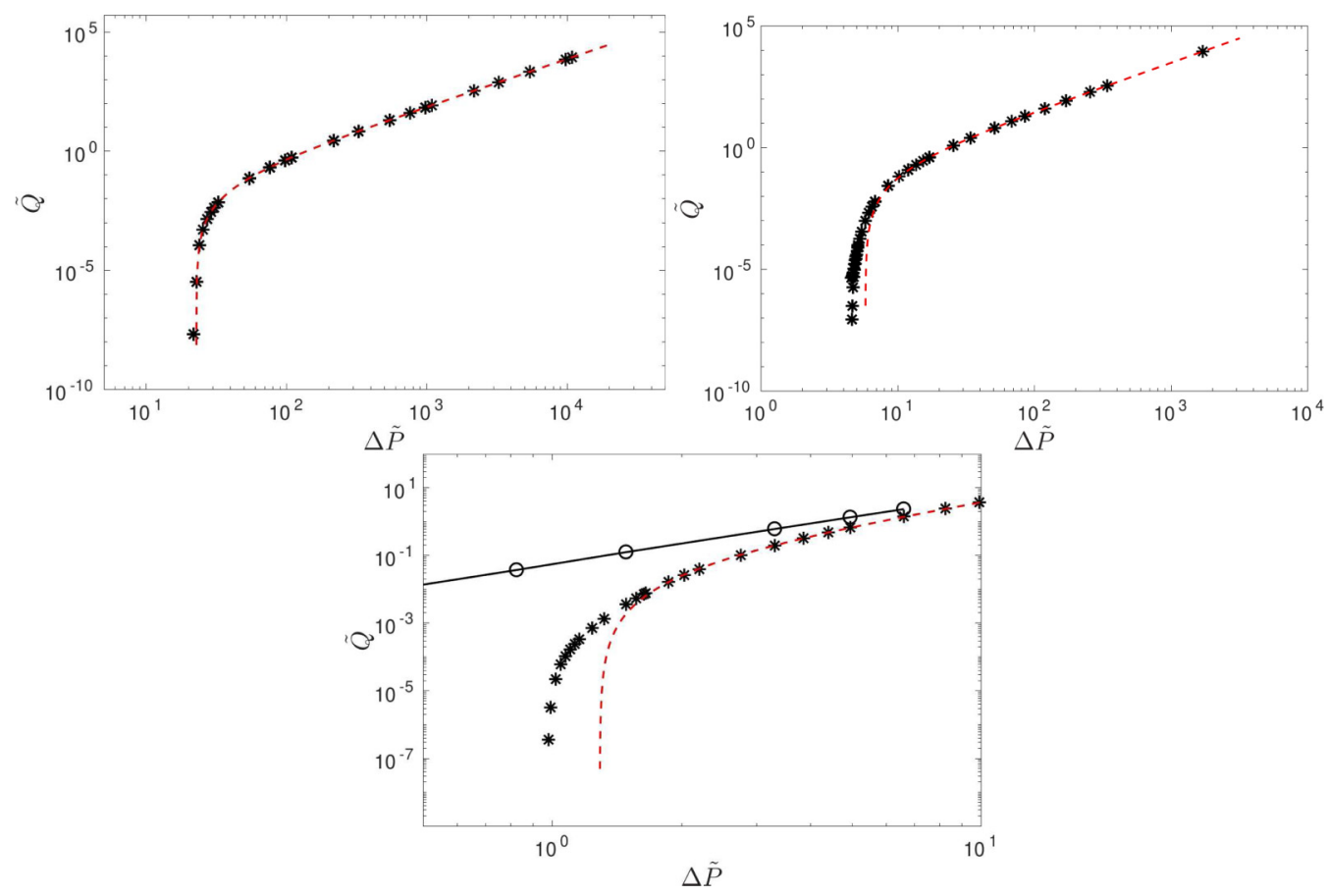

FIG. 4. Numerical flow rate as function of the nondimensional pressure drop. Top left: FCC; Top right: irregular bead packing; Bottom: sandstone. Stars represent numerical data, and dashed lines represent the fit with Eq. (12) considering only the high flow rate. The open circles correspond to simulations of a Newtonian fluid $\left(\tau_{0}=0\right)$.

Eq. (2) in the following form:

$$
\tilde{Q}^{n}=A\left(\Delta \tilde{P}-\Delta \tilde{P}_{c}^{\infty}\right)
$$

by setting a weight on the higher values of $\tilde{Q}$. We imposed $n=0.53$ obtained from rheological measurements with the tube. The best fit for the higher flow rate is rather good within an error of $\lesssim 10^{-3}$. The reason why we only consider the higher flow rate is because one cannot achieve such a small error with the whole data using this fitting function. The best fit at higher rates implies a significant discrepancy of the experimental data at lower rate. In particular, the pseudocritical pressure $\tilde{P}_{c}^{\infty}$ is larger than the true critical pressure which means that the fluid is indeed flowing at lower pressure than the one predicted by the LPG model.

\section{B. Simulation by LB-TRT}

For the simulations we chose the following parameters for the fluid rheology: $\tau_{0}=10^{-3}, k=$ $10^{-3}$, and $n=0.5$ (in lattice units). Figure 4 shows $\tilde{Q}$ as a function of $\Delta \tilde{P}$ for the different porous structures and the fit using Eq. (12) with $n=0.5$. For the irregular sphere packing and particularly for the sandstone, results are similar to those obtained experimentally: the fit is quite good (error $\lesssim 10^{-2}$ ) if we restrict it to higher flow-rate data. An important difference between numerical data and the fit is then observable for lower flow rates. The threshold pressure of the numerical data is also much lower than the one obtained by the fit. However, it is important to note that for FCC, the LPG model, Eq.(12), fits quite well the numerical data even at lower flow rate (error $\sim 10^{-2}$ ). This supports the contention that the difference between the true and pseudo critical pressure is related to the disorder of the medium. 
TABLE I. Values of the "true" and "pseudo" pressure thresholds.

\begin{tabular}{lcc}
\hline \hline Porous structure & $\Delta \tilde{P}_{c}$ & $\Delta \tilde{P}_{c}^{\infty}$ \\
\hline FCC & 22.8 & 22.8 \\
Irregular packing & 4.59 & 5.79 \\
Sandstone (simulation) & 0.97 & 1.29 \\
Sandstone (exp) & 1.6 & 2.58 \\
\hline \hline
\end{tabular}

\section{Determination of the true critical pressure threshold $\Delta \tilde{\boldsymbol{P}}_{c}$}

The determination of the true critical pressure $\Delta \tilde{P}_{c}$ is based on Eq. (3) but by focusing on the lower-flow-rate data. The fit must, however, be performed differently. The main reason is that we do not know a priori the exponent $\alpha_{i}$ at the true critical pressure. We used the fact that the flow rate must tend to zero when the pressure reaches the critical pressure. Namely, we plot the function $\tilde{Q}=f\left(\Delta \tilde{P}-\Delta \tilde{P}_{c}\right)$ on a log-log scale and vary $\Delta \tilde{P}_{c}$ until the curve becomes a straight line in the limit $\tilde{Q} \rightarrow 0$ and $\Delta \tilde{P}-\Delta \tilde{P}_{c} \rightarrow 0$, characterizing a power-law.

Values of $\Delta \tilde{P}_{c}$ and $\Delta \tilde{P}_{c}^{\infty}$ are given in Table I. As can be seen values of the true pressure threshold are smaller than the pseudo pressure threshold except for FCC.

\section{DISCUSSION}

\section{A. Significance of the pressure threshold: True pressure versus pseudopressure threshold}

In a previous work, Talon and Bauer [1] have shown numerically in a $2 \mathrm{D}$ porous medium that $\Delta \tilde{P}_{c}$ corresponds to the minimal pressure required for the onset of the flow in a single channel. Here, Fig. 5. shows the streamlines in the 3D random packing for different pressure drops. As can be seen, for values close to $\Delta \tilde{P}_{c}$ the fluid is flowing in only one channel. With increasing pressure the number of open channels increases. This reflects the occurrence of preferential paths introduced by the disorder of the heterogeneous structures which are easier to yield. In contrast to the latter, for FCC we obtain $\Delta \tilde{P}_{c}=P_{c}^{\infty}$. This stands for the fact that in the homogeneous structure fluid is flowing in the entire porous medium once the critical pressure threshold is reached because all paths are equivalent. The difference between the "true" and "pseudo" critical pressure threshold can be formally explained by means of an energy balance.

\section{Energy balance}

Following Chevalier et al. [11], at imposed pressure difference, one can relate the flow rate to the viscous and plastic dissipation in the medium (see also Appendix D for details):

$$
\Delta P Q(\vec{u})=\int\left[k \dot{\gamma}^{n+1}(\vec{u})+\tau_{0} \dot{\gamma}(\vec{u})\right] d v,
$$

where $Q(\vec{u})$ is the mean flow rate and $\dot{\gamma}(\vec{u})$ the shear rate field.

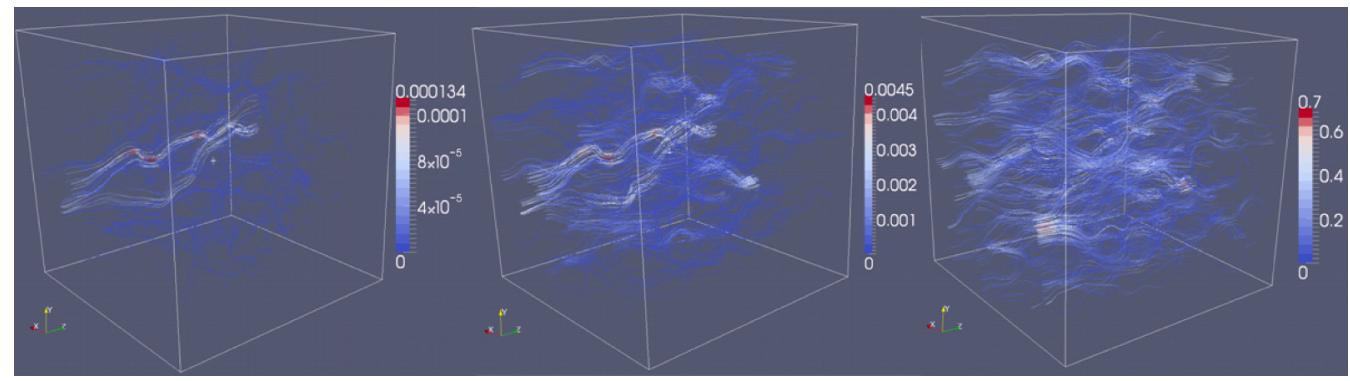

FIG. 5. Streamlines inside a random packing of spheres for increasing applied pressure drop. 
Introducing the nondimensional shear rate distribution $g_{u}(\vec{r}, \Delta P)=\frac{S \lambda}{Q(\vec{u})} \dot{\gamma}(\vec{u})$, it follows

$$
\Delta P=k \frac{Q^{n}(\vec{u}) L}{S^{n} \lambda^{n+1}}\left\langle g_{u}^{n+1}\right\rangle+\frac{\tau_{0} L}{\lambda}\left\langle g_{u}\right\rangle
$$

or

$$
\frac{\Delta P}{L}=A_{u} Q^{n}+B_{u},
$$

where $\langle$.$\rangle stands for the volume average \frac{1}{S L} \int . d v, A_{u}=\frac{k}{S^{n} \lambda^{n+1}}\left\langle g_{u}^{n+1}(\vec{r}, \Delta P)\right\rangle$ and $B_{u}=$ $\frac{\tau_{0}}{\lambda}\left\langle g_{u}(\vec{r}, \Delta P)\right\rangle$.

This relation leads to an expression of the flow rate as well as of the critical pressure providing the knowledge of the two moments of the shear rate distribution. It is important to emphasize that, $A_{u}$ and $B_{u}$ are not constant as it is the case in Eq. (2). Indeed, moments of the shear rate distribution are a priori a function of the applied pressure. Their determination therefore requires to solve the full Stokes equation.

\section{Functional minimization}

Alternatively, one might deduce some properties of $g_{u}(\vec{r}, \Delta P)$ via a variational principle [26], which is a generalization of the well-known minimum energy dissipation of Stokes flows [27]. Namely, at imposed pressure difference, it states that the flow field $\vec{u}$, solution of the Stokes equation for Herschel-Bulkley fluid, minimizes the following functional (see Appendix D):

$$
\begin{aligned}
\Phi[\vec{v}, \Delta P]= & \frac{1}{n+1} k \frac{Q^{n+1}(\vec{v}, \Delta P)}{S^{n+1} \lambda^{n+1}} \int g_{v}^{n+1}(\vec{r}, \Delta P) d r^{3} \\
& +\frac{\tau_{0}}{S \lambda} Q(\vec{v}, \Delta P) \int g_{v}(\vec{r}, \Delta P) d r^{3}-\Delta P Q(\vec{v}, \Delta P),
\end{aligned}
$$

where $\vec{v}$ is taken among all the admissible velocity fields (i.e., satisfying specific boundary conditions defined in Appendix D and the free divergence). It is worth mentioning that this functional differs from the viscous and plastic dissipation Eq. (13).

The comparison between the first and second term in Eq. (16) allows us to distinguish two limiting cases.

(1) Low-flow-rate regime $Q \ll\left(\frac{\tau_{0}}{k}\right)^{1 / n} S \lambda$

In this case, the first term in Eq. (16) is negligible compared to the second one. The function reduces to

$$
\Phi[\vec{v}, \Delta P]=Q(\vec{v}, \Delta P)\left[\frac{\tau_{0}}{S \lambda} \int g_{v}(\vec{r}, \Delta P) d r^{3}-\Delta P\right],
$$

which defines the "true" critical pressure formally as

$$
\Delta P_{c}=\min _{\vec{v}} \frac{\tau_{0}}{S \lambda} \int g_{v}(\vec{r}, \Delta P) d r^{3},
$$

where we have used the fact that when $\Delta P<\Delta P_{c}$ the term in brackets in Eq. (17) is positive for any nontrivial velocity field. The minimum of $\Phi$ is then the trivial one: $\vec{v}=\overrightarrow{0}, Q=0$ and $\Phi(\vec{v}, \Delta P)=0$.

(2) High-flow-rate regime $Q \gg\left(\frac{\tau_{0}}{k}\right)^{1 / n} S \lambda$

Here the second term in Eq. (16) becomes negligible compared to the first one. The problem reduces to minimizing the functional

$$
\Phi^{\mathrm{PL}}[\vec{v}, \Delta P]=\frac{1}{n+1} k \frac{Q^{n+1}(\vec{v}, \Delta P)}{S^{n+1} \lambda^{n+1}} \int g_{v}^{n+1}(\vec{r}, \Delta P) d r^{3}-\Delta P Q(\vec{v}, \Delta P) .
$$

As can be seen, the latter equation is independent of $\tau_{0}$ (or $\tau_{0}=0$ ), the problem is thus identical to solving a power-law fluid (or Newtonian flow when $n=1$ ) which results in the shear rate 
distribution $g_{u}^{\mathrm{PL}}(\vec{r}, \Delta P)$ (PL stands for power law). This result should be compared to the approach of Chevalier et al. [11], which used the knowledge of the shear rate distribution of a power-law fluid to determine the coefficients $A_{u}$ and $B_{u}$ in the generalized Darcy's law of a yield stress fluid. More specifically the authors used the shear rate distribution of a Newtonian fluid $(n=1)$ to compute the coefficients. This assumption might be formally difficult to justify. However, the consistency of their experimental results with this assumption could be interpreted from the fact the moments of order 2 and $n+1 \simeq 1.5$ used to minimize Eq. (19) are relatively close.

At this point, we may make an additional comment regarding the dependence of the coefficients $A_{u}$ and $B_{u}$ on the applied pressure drop. We note that the determination of the minimum of Eq. (19) is homogeneous with respect to $\Delta P$. Indeed, for any $\alpha$, multiplying by $\alpha^{1 / n+1}$, we have

$$
\begin{aligned}
\arg & \min _{v} \frac{1}{n+1} k \frac{Q^{n+1}(\vec{v}, \Delta P)}{S^{n+1} \lambda^{n+1}} \int g_{v}^{n+1}(\vec{r}, \Delta P) d r^{3}-\Delta P Q(\vec{v}, \Delta P) \\
= & \arg \min _{v} \frac{1}{n+1} k \frac{\left[\alpha^{1 / n} Q(\vec{v}, \Delta P)\right]^{n+1}}{S^{n+1} \lambda^{n+1}} \int g_{v}^{n+1}(\vec{r}, \Delta P) d r^{3}-(\alpha \Delta P)\left[\alpha^{1 / n} Q(\vec{v}, \Delta P)\right] .
\end{aligned}
$$

The flow field solution is thus identical for any rescaling of the imposed pressure $\alpha \Delta P$. It follows that the flow rate in this regime is a homogeneous function of degree $1 / n: Q^{\mathrm{PL}}(\alpha \Delta P)=$ $\alpha^{1 / n} Q^{\mathrm{PL}}(\Delta P)$ and thus $Q(\Delta P) \propto(\Delta P)^{1 / n}$. We deduce that at high $Q$, when all the fluids is flowing, the normalized shear rate field, $g_{u}^{P L}(\vec{r})$, is invariant of the applied pressure. $A_{u}$ and $B_{u}$ are thus independent of $Q$ or $\Delta P$, leading to the generalized Darcy's law:

$$
\frac{\Delta P}{L}=A Q^{n}+\frac{\Delta P_{c}^{\infty}}{L}
$$

where $A$ is a constant and $\Delta P_{c}^{\infty}$ is the "pseudo" critical pressure defined by

$$
\Delta P_{c}^{\infty}=\frac{\tau_{0}}{S \lambda} \int g^{P L}(\vec{r}) d r^{3} .
$$

We note that based on the expressions of the "true" and "pseudo" critical pressure [Eqs. (18) and (22)], we deduce $\Delta P_{c} \leqslant \Delta P_{c}^{\infty}$. The equality is achieved when the two distributions, resulting from the minimization of different functionals, have the same first moment.

Finally, we may derive identical conclusions if we consider flow induced by an imposed flow rate $Q$. Indeed, one can show (see Appendix D for details) that the flow field minimizes the functional:

$$
\Phi[\vec{v}, Q]=\frac{1}{n+1} k \frac{Q^{n+1}}{S^{n+1} \lambda^{n+1}} \int g_{v}^{n+1}(\vec{r}, Q) d r^{3}+\frac{\tau_{0}}{S \lambda} Q \int g_{v}(\vec{r}, Q) d r^{3},
$$

which defines the same "true" and "pseudo" critical pressure.

\section{B. Disorder and macroscopic scaling law: Flow rate versus distance to the critical pressure}

A major evidence of the effect of even slight disorder occurs when we substract the true critical pressure, obtained using the procedure described in Sec. III C, from the imposed pressure. Figure 6 displays the nondimensional flow rate $\tilde{Q}$ as function of the distance of the pressure to the measured critical pressure (experimental and numerical results). By doing so, we deduce a common law between the flow rate and the applied pressure under the following form: $\tilde{Q}$ versus $\left(\Delta \tilde{P}-\Delta \tilde{P}_{c}\right)^{1 / n}$, which demonstrates the existence of at least three flowing regimes. While for the FCC packing, the flow curve displays a single power-law behavior of exponent $1 / n=2$, the other curves show a different behavior. For the irregular packing the curve initially starts with a power-law of exponent 2 , then there is an intermediate regime for $\tilde{Q} \in\left[10^{-5} ; 10^{-1}\right]$. We have fit this regime with a power law $\tilde{Q} \propto\left(\Delta \tilde{P}-\Delta \tilde{P}_{c}\right)^{3}$. Finally, as mentioned above, the last regime corresponds to the power-law fluid limit $\tilde{Q} \propto \Delta \tilde{P}^{1 / n}$.

As it is the case for the random packing, the numerical flow curve of the sandstone displays three power-law behaviors. The exponents $1 / n$ are successively equal to $2.0,2.6$, and 2.0. It is worth 

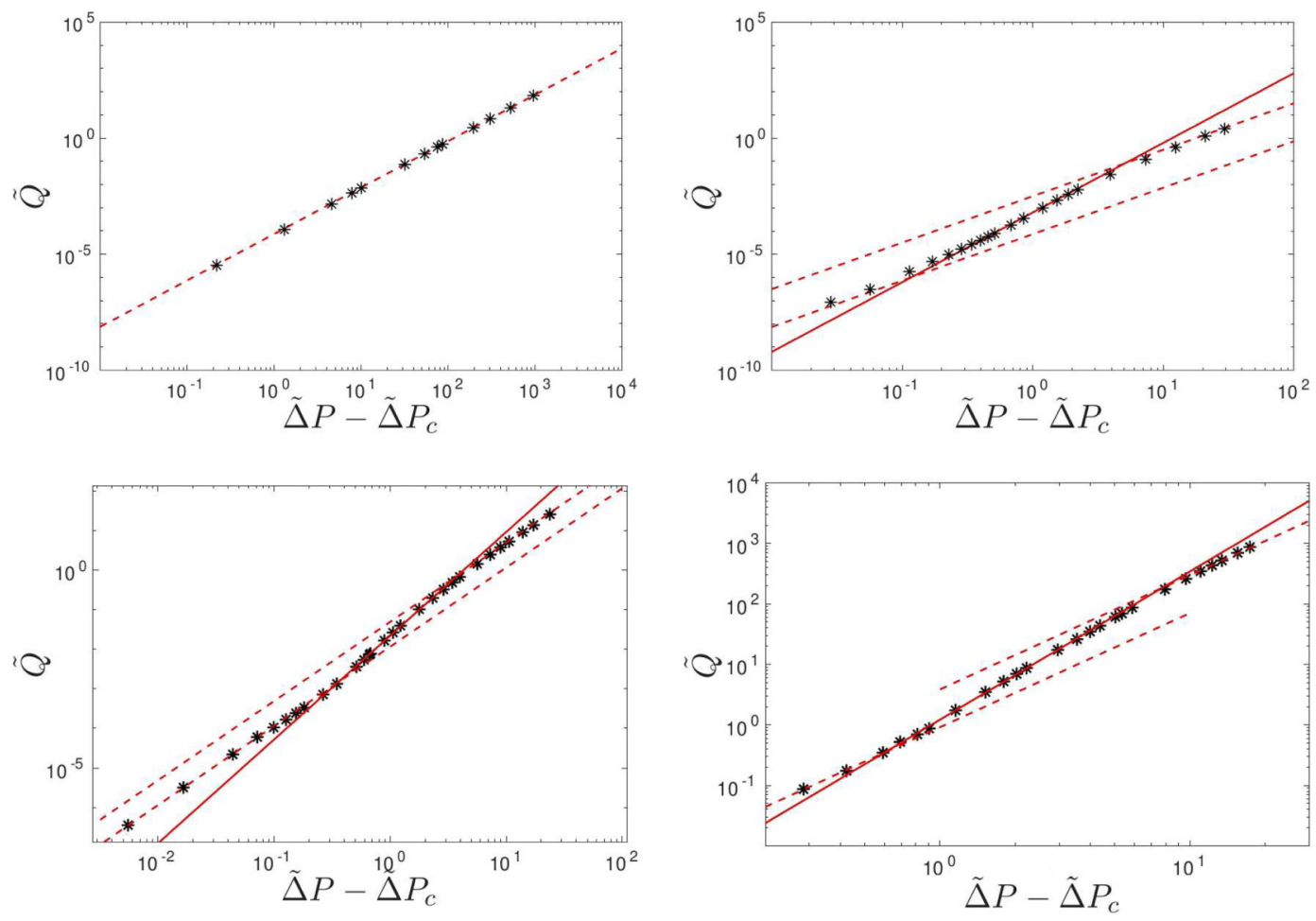

FIG. 6. Top left: FCC sphere packing; top right: random sphere packing; bottom left: sandstone simulation; bottom right: sandstone experiments. Stars correspond to numerical or experimental data, and dashed and solid lines correspond to the power-law fit. (Please refer to the text for values of the exponents.)

mentioning that the exponent in the intermediate regime is different for the random packing and the sandstone. This contradicts the results of the 2D simulations which led to the same exponent for different disorder. We note, however, that the range of flow rate of the intermediate regime is relatively small, which makes the measurement of the exponent of this regime quite imprecise.

As it has been discussed in Ref. [17], the range of the second regime can be related to the size of the system. In particular, it depends on the ratio of the pore size to the system size. One natural way to increase the range of the second regime and thus the precision would be to increase the system size. Unfortunately, this could not be achieved with our numerical resources.

Considering the experimental data we also obtain three regimes where the exponents successively equal to $1.89,2.45$, and 1.89 . Interestingly, we note that despite the difference of the rheological index between the simulation $(n=0.5)$ and experiments $n=0.53$, the increase between the first and second exponent is comparable.

Despite the exponents' values, these flow regimes are identical to the flow regimes observed by Refs. $[1,17]$ in 2D porous media. The fact that these regimes occur only in the random packing and the sandstone supports the contention that they are the consequence of the disorder of the medium. Indeed, even in homogeneous, however slightly disordered porous structures as sandstones, the three regimes can be observed.

\section{CONCLUSION}

In this work, we investigated numerically and experimentally Herschel-Bulkley fluid flow in porous media of different structure. To this goal, we performed 3D Lattice Boltzmann simulations using a TRT scheme and developed a specific experimental set-up allowing the fluid to flow in a 
closed-loop system. Initially, fluid properties are modified when flowing in the porous medium, however after a transient regime, fluid rheology remains stationary. The closed-loop system permits measurements in the stationary regime necessary for the determination of a generalized Darcy equation.

We have shown that for three-dimensional heterogeneous structures, identically as for $2 \mathrm{D}$ structures, a "true" critical pressure $\tilde{P}_{c}$ exists, that is lower than the "pseudo" threshold pressure $\tilde{P}_{c}^{\infty}$ commonly used in LPG models. In the homogeneous porous medium (FCC) we obtained $\tilde{P}_{c}=\tilde{P}_{c}^{c}$, however in the heterogeneous structures we have $\tilde{P}_{c}<\tilde{P}_{c}^{\infty}$. Additionally, for the sandstone and the irregular sphere packing we observed numerically and experimentally the three scaling regimes when plotting the flow rate $\tilde{Q}$ as a function of the difference of the pressure to the critical pressure $\left(\Delta \tilde{P}-\Delta \tilde{P}_{c}\right)$. However, for the FCC packing the LPG model describes perfectly the flow-rate-pressure behavior.

We explained this difference in critical pressure inducing the existence of the scaling regimes by means of an energy minimization principle. Indeed, it states that the first opening pathway in a heterogeneous porous medium is characterized by the minimal energy loss of the fluid when flowing in this structure.

In the Appendices, we present in detail measurements of the Carbopol rheology, the TRT Lattice Boltzmann method and its validation in 3D structures, and the analytical determination of the critical pressure.

\section{ACKNOWLEDGMENT}

The authors thank the Agence National de la Recherche for financial support of the project LaboCothep (Grant No. ANR-12-MONU-0011).

\section{APPENDIX A: CARBOPOL GELS-PREPARATION AND RHEOLOGY}

Carbopol ETD 2050, kindly provided by Lubrizol, was chosen in the present work. The preparation starts with the introduction of some distilled water in a mixer. The mixing rotation velocity is set to $90 \mathrm{rpm}$ and the appropriate amount of raw Carbopol powder is slowly added to the stirring water. After about 2-4 h, depending on the final quantity of the gel, the incorporation of the powder is done and the $\mathrm{pH}$ drops to a value of 3-4. Then, an appropriate amount of sodium hydroxide $(\mathrm{NaOH} 1 \mathrm{~mol} / \mathrm{l})$ is quickly added to the solution. The $\mathrm{pH}$ of the solutions is set to 7.0 (with 0.2 error bar). With a pH range between 6 and 8, it has been shown that the rheological properties of Carbopol gels are stable [19]. The mixing is then maintained for 1 day to allow a full homogenization of the mixture. Such a procedure makes it possible to obtain a reproducible material which remains stable in time. The gels were kept away from light in a refrigerator at a temperature of $4{ }^{\circ} \mathrm{C}$.

Rheological tests were performed with a MCR 300 rheometer equipped with a Mooney-Ewart (or Searle) cylinder. The sample was carefully set up and the gap was fixed at $0.6 \mathrm{~mm}$ taking care not to entrap air bubbles. The measurements were performed by imposing a range of shear rate, $\dot{\gamma}$, from $10^{-6} \mathrm{~s}^{-1}$ to $10^{-3} \mathrm{~s}^{-1}$ in logarithmically increasing and decreasing plateaus. Except for the first part of the increasing curve associated with deformations in the solid regime, the increasing and decreasing shear-stress versus shear-rate curves almost superimpose. A hysteresis phenomenon or the thixotropic nature of Carbopol is observed when the applied force is lower than the critical stress $\left(\tau_{0}\right)$ : the return to the solid state involves a relaxation time. We retained the decreasing part of the flow curve to measure the rheological fluid parameters of the Herschel Bulkley model.

\section{APPENDIX B: EXPERIMENTAL VALIDATION FOR A NON-NEWTONIAN FLUID}

Figure 7 shows the rheology obtained before and after the experiments by means of a rheometer as well as the data obtained from measurements in the reference tube once the pressure was stabilized.

Table II shows the rheological parameters obtained by the different measurements. 


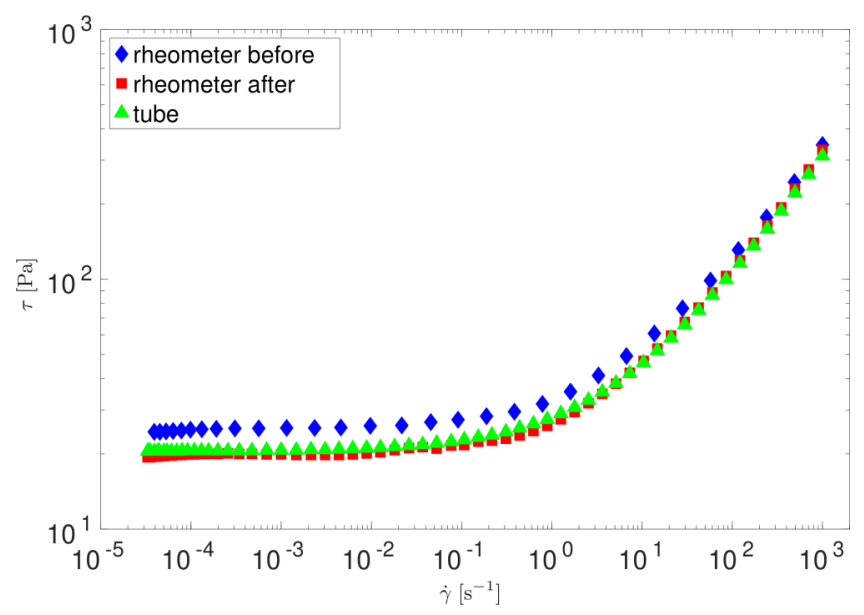

FIG. 7. Carbopol rheology obtained before and after the experiments by means of a rheometer and data obtained from the reference tube.

\section{APPENDIX C: NUMERICAL METHOD}

\section{A brief of the TRT scheme}

The principle of the lattice Boltzmann (LB) method for solving Stokes equation is based on a discretization of the velocity distribution function of particles on a grid. For this purpose, $f_{q}$ is introduced as the density of particles moving with the velocity $\boldsymbol{c}_{q}$. The numerical algorithm of LB methods is a succession of two steps. (i) The propagation step, where the particle density is moved on the grid according to their velocity:

$$
f_{q}\left(\boldsymbol{r}+\boldsymbol{c}_{q}, t+1\right)=\tilde{f}_{q}(\boldsymbol{r}, t) .
$$

(ii) The second step is the collision step, where in each node of the grid, the velocity distribution of particles is redistributed using a collision operator $\boldsymbol{C}$ which depends on the local macroscopic quantities (pressure, velocity,...):

$$
\tilde{f}_{q}(\boldsymbol{r}, t)=\boldsymbol{C}\left[f_{q}(\boldsymbol{r}, t)\right]_{q} .
$$

In this paper, we used a three-dimensional scheme with 15 velocities (D3Q15), $\boldsymbol{c}_{q}, q=1, . ., 15$. To compute the collision operator, populations are arranged such that the first 7 vectors are opposite to the second set of 7 other vectors, defined as $\boldsymbol{c}_{\bar{q}}=-\boldsymbol{c}_{q}$. For the two-relaxation-times (TRT) scheme, the population distribution is decomposed in symmetric components $f_{q}^{+}=\left(f_{\bar{q}}+f_{q}\right) / 2$, and antisymmetric components $f_{q}{ }^{-}=\left(f_{\bar{q}}-f_{q}\right) / 2$. For immobile population, the velocity is defined as $\boldsymbol{c}_{q}=0$, and the density function is $f_{0}{ }^{+}=f_{0}$ and $f_{0}{ }^{-}=0$. The collision step is performed by prescribing the equilibrium distributions $e_{q}{ }^{ \pm}$and two collision eigenvalues $\left.s^{ \pm} \in\right] 0,2\left[\right.$, with $s^{+}$for all symmetric and $s^{-}$for all antisymmetric nonequilibrium components, $n^{+}$and $n^{-}$, respectively.

TABLE II. Rheological properties measured before and after flowing in the porous media at various flow rates.

\begin{tabular}{lccr}
\hline \hline & $\tau_{0}[\mathrm{~Pa}]$ & $k\left[\mathrm{~Pa} \mathrm{~s}{ }^{n}\right]$ & $n[]$ \\
\hline Tube & 20.5 & 7.5 & 0.53 \\
Rheometer before & 24.5 & 9.5 & 0.51 \\
Rheometer after & 19.2 & 7.5 & 0.54 \\
\hline \hline
\end{tabular}


We obtain the following relations:

$$
\begin{aligned}
& \tilde{f}_{q}(\boldsymbol{r}, t)=f_{q}(\boldsymbol{r}, t)-s^{+} n_{q}{ }^{+}-s^{-} n_{q}{ }^{-}, \\
& \tilde{f}_{\bar{q}}(\boldsymbol{r}, t)=f_{q}(\boldsymbol{r}, t)-s^{+} n_{q}{ }^{+}+s^{-} n_{q}{ }^{-},
\end{aligned}
$$

with the nonequilibrium components

$$
n_{q}{ }^{ \pm}=f_{q}^{ \pm}-e_{q}{ }^{ \pm}
$$

The equilibrium functions $e_{q}{ }^{ \pm}$of the Stokes equation are

$$
\begin{aligned}
& e_{q}^{+}=c_{s}^{2} t_{q}^{*} \rho, \\
& e_{q}^{-}=t_{q}^{*}\left(\boldsymbol{j} \cdot \boldsymbol{c}_{q}\right), \\
& e_{0}=\rho-2 \sum_{q=1}^{7} e_{q}^{+},
\end{aligned}
$$

where the weights $t_{q}^{*}$ take the values $1 / 3$ for the first neighbor link and $1 / 24$ for the second (diagonal) neighbor link in the D3Q15 model.

The TRT scheme solves Stokes equation with the kinematic viscosity, given by $v_{\mathrm{eff}}=\frac{1}{3}\left(\frac{1}{s^{+}}-\frac{1}{2}\right)$, the pressure $P$ is defined as $P=c_{s}^{2} \rho$, and the macroscopic momentum $\boldsymbol{u}=\boldsymbol{j} / \rho_{0}$, where $\rho_{0}$ is the mean density of the fluid $\left(\rho_{0}=1\right.$, here). The sound velocity is set to $\sqrt{1 / 3}$, and the relaxation parameter $\Lambda=\left(\frac{1}{s^{+}}-\frac{1}{2}\right)\left(\frac{1}{s^{-}}-\frac{1}{2}\right)$ is kept constant $(\Lambda=3 / 16)$. The fluid takes initially a homogeneous effective viscosity $\nu_{\text {eff }}$, but it varies with the local shear rate $\dot{\gamma}$. The local deformation rate tensor is obtained from the nonequilibrium components:

$$
\boldsymbol{\Delta}_{i j}=\frac{1}{2 \rho_{0}}\left(\nabla \boldsymbol{j}+\nabla \boldsymbol{j}^{T}\right)=-s^{+} \frac{3}{2 \rho_{0}} \sum_{1}^{15} n_{q}^{+} c_{q i} c_{q j}=-s^{+} \frac{3}{\rho_{0}} \sum_{1}^{7} n_{q}^{+} c_{q i} c_{q j} .
$$

The TRT algorithm is implemented as follows. After taking an initial value of a homogeneous effective viscosity $v_{\text {eff }}$, the pressure gradient is applied between the inlet and the outlet of the sample. For each time step, the shear rate at each node of the grid is computed using Eq. (C5) to update the local viscosity following Eq. (11). The convergence is reached when the difference of the flow field between two iterations is lower than a typical criteria $\left(\varepsilon<10^{-3}\right.$ in our code). This might take around $10^{4}$ and $10^{9}$ time steps, depending on the size of the microstructures.

\section{Validation of the TRT code and influence of the grid resolution}

Because the resolution of the $\mu$-CT images is limited by the microtomography, we want to estimate the error induced by the rather low grid resolution in different type of geometries.

\section{a. Cylindrical Poiseuille flow}

The velocity field of a Herschel-Bulkley fluid in a cylindrical tube is known analytically and is given by

$$
\begin{aligned}
& \text { for } r \leqslant r_{c}: v_{\max }=\frac{n}{n+1}\left(\frac{1}{2 k} \frac{\Delta P}{L}\right)^{1 / n}\left(R-r_{c}\right)^{1+\frac{1}{n}}, \\
& \text { for } r \geqslant r_{c}: v(r)=v_{\max }\left(1-\left(\frac{r-r_{c}}{R-r_{c}}\right)^{1+\frac{1}{n}}\right),
\end{aligned}
$$

where $r_{c}=\frac{2 \tau_{0} L}{\Delta P}$ is the radius at which the yield stress is reached. 

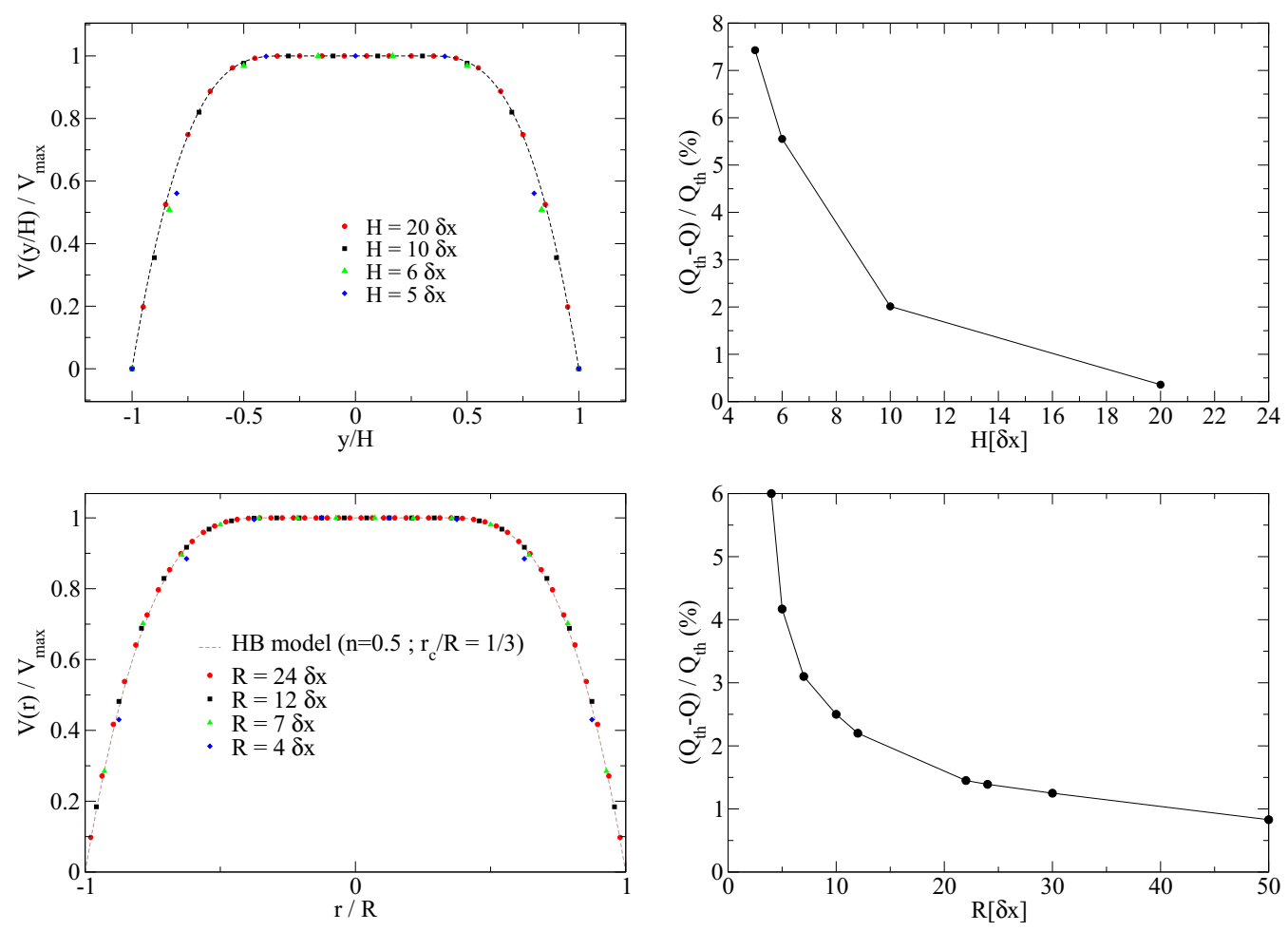

FIG. 8. Comparison of the numerical and theoretical velocity profile (left) and flow rate (right) (top: parallel planes; bottom: 3D cylindrical tube).

The flow rate is given by

$$
Q=\frac{\pi R^{3}}{k^{1 / n}(1 / n+3)} \tau_{w}^{1 / n}\left(1-\frac{\tau_{0}}{\tau_{w}}\right)^{1+\frac{1}{n}}\left[1+A \frac{\tau_{0}}{\tau_{w}}+B\left(\frac{\tau_{w}}{\tau_{0}}\right)^{2}\right],
$$

with

$$
\begin{aligned}
& A=\frac{2}{2+\frac{1}{n}} \\
& B=\frac{2}{\left(2+\frac{1}{n}\right)\left(1+\frac{1}{n}\right)} .
\end{aligned}
$$

Velocity profile and flow rate between two parallel planes can be calculated identically.

Simulations are performed between two parallel planes and in a $3 \mathrm{D}$ cylindrical tube and compared to the theoretical velocity profiles and flow rates (see Fig. 8). Velocity profiles are in very good agreement with the theoretical prediction [Eq. (C7)]. Also, the difference between the theoretical flow rate [Eq. (C8)] and the numerically computed flow rate remains small even for the very coarse grid.

\section{b. Sinusoidal 2D channel}

Cylindrcal tubes and parallel plates are not very representative of real porous media. We therefore investigate flow in a channel with spatially varying aperture as in Refs. [28,29]. We consider the same $2 \mathrm{D}$ wavy channel whose aperture follows a cosine function between the $2 \mathrm{R}$ and $2 \mathrm{R}+2 \mathrm{H}$ values. 

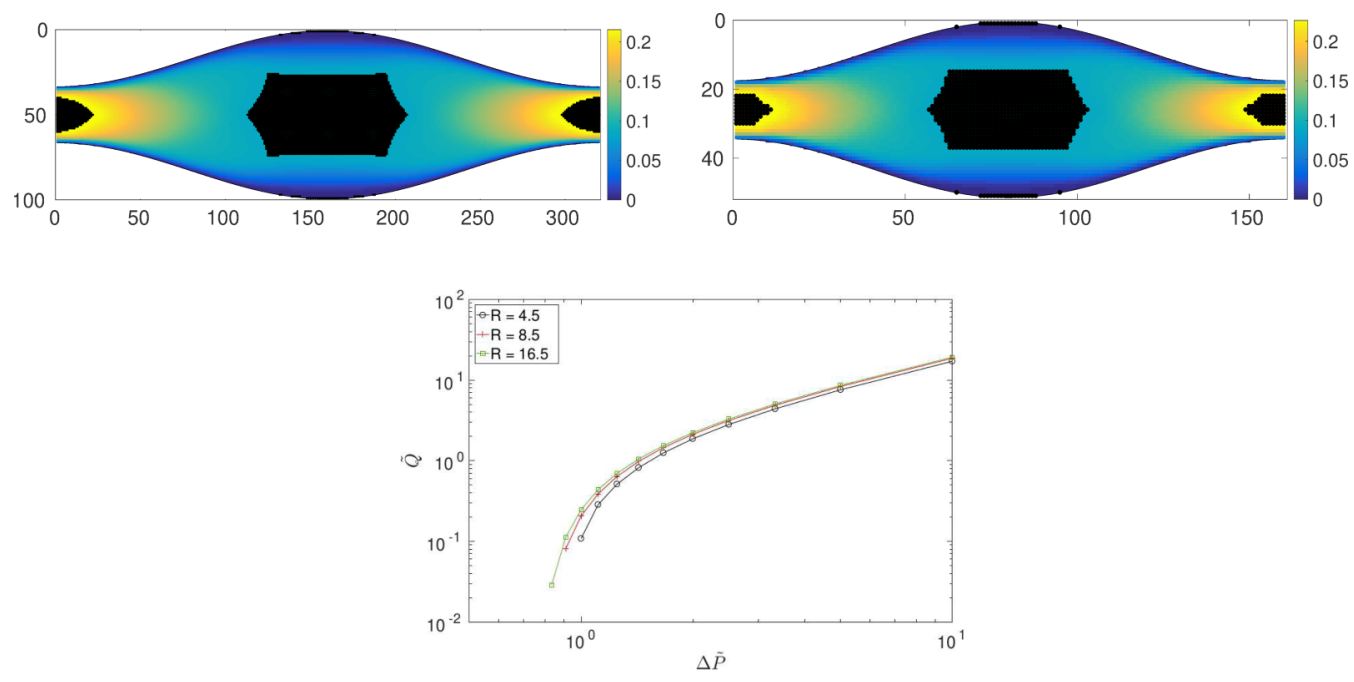

FIG. 9. Top: flow field in a wavy cosine channel for two different resolution (left: $R=16.5$; right $R=8.5$ ). The black dots represent the unyielded regions. Bottom: flow rate as function of the pressure drop for different resolution.

We perform flow simulations for a Bingham rheology $(n=1)$ using different resolutions with $R=4.5,8.5$ and 16.5 voxels, $R$ being the radius of the restriction. As shown in Fig. 9, all simulations capture the main effect of the change in aperture on the flowfield, which is to break the central plug due to the elongation rate. As we have shown in the previous section, in the center of a straight channel, there is always a region where the plug has not yielded. But when the aperture varies, the conservation of the flow rate induces a rate of elongation that tends to unyield the fluid.

In addition, with regard to the flow in the channel, Fig. 9 shows that the flow curve remains relatively accurate even for a very coarse grid. The difference between the critical pressure between high and low resolution is only about $10 \%$.

Moreover, in this figure it can be seen that each resolution leads to a specific translation of the curve but not to a significant modification of its shape. As a result, the numerical error mainly affects the critical pressure but not the overall physics. The following qualitative interpretation is based on Refs. [22,30], indicating that one of the main sources of error in the TRT scheme is the uncertainty about the actual position of the wall between two voxels. The flow curve of each resolution therefore corresponds to a channel whose width is slightly different from that expected.

\section{c. FCC packing}

We now investigate the influence of the resolution on the flow in the FCC packing. In Fig. 10, we plot the the flow curve for different bead resolutions, $R=14,28$, and 56 voxels. It can be seen that the flow curve, while maintaining a similar trend, is also shifted by a factor of about $15 \%$ depending on the resolution.

In conclusion of this resolution study, it can be said that one of the main advantages of the TRT scheme is its robustness. Even at low resolution, simulations lead to physically reasonable solutions. Estimating an equivalent pore diameter for an actual rock structure is very difficult. However, considering the flow field near the critical pressure (Fig. 5), it can be estimated that the the average streamtube diameter is about 15 voxels, which represents a numerical error of about $15 \%$ for the critical pressure. 


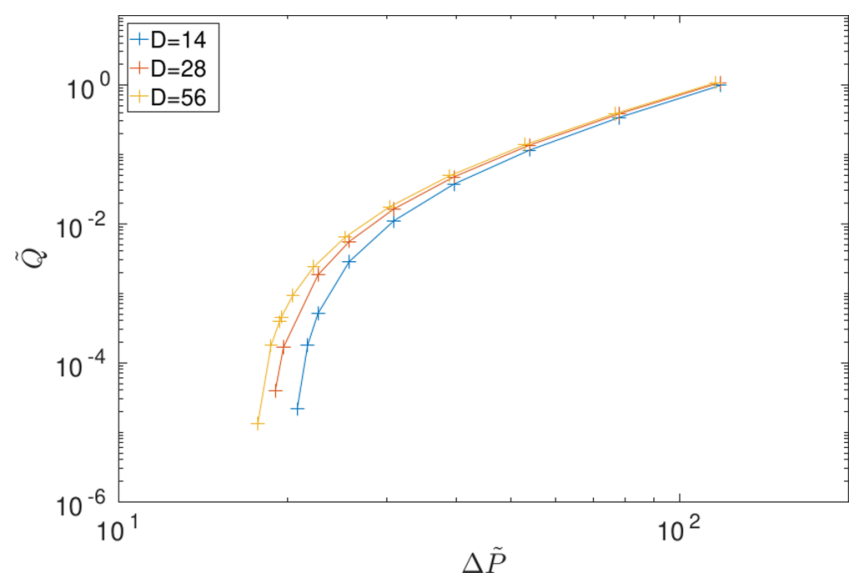

FIG. 10. Flow rate as function of the pressure drop for different resolution of FCC packing.

\section{Validation of the regularization}

Figure 11(a) shows the flow rate $Q$ of the FCC packing as a function of the pressure difference $\Delta P$ for different values of $\tau_{0}$. Figure 11(b) represents the nondimensionalized flow curves. It can be seen that the curves superimpose on the same master curve except some points for $\tau_{0}=10^{-6}$ for low $\tilde{\Delta} P$. The good collapse demonstrates that the numerical TRT scheme is correctly invariant of the effective viscosity, as opposed to the classical BGK. This curve also points out the significance of the numerical parameter $\dot{\gamma}_{0}$. The fact that some points for the low yield stress value $\tau_{0}=10^{-6}$ do not coincide with the master curve is a consequence of the regularization technique and the choice of the different parameters. As described before, $\dot{\gamma}_{0}$ represents the shear rate below which the regularization will be observable. However, if the shear rate is much higher than yield stress $\left(\frac{\tau_{0}}{k}\right)^{1 / n}$, then the role of plasticity becomes negligible. It exists thus a bounded range of flow rates over which the effect of plasticity is apparent. This requires us to choose the numerical parameters such that $\dot{\gamma}_{0} \ll\left(\frac{\tau_{0}}{k}\right)^{1 / n}$. As described in the manuscript, the different flow regimes involve at least six orders of magnitude for the flow rate. We used the set $\left[k ; \tau_{0} ; \dot{\gamma}_{0}=\left(10^{-3} ; 10^{-3} ; 10^{-9}\right)\right.$ in lattice units], which permits a range of nine orders of magnitude for the flow rate.
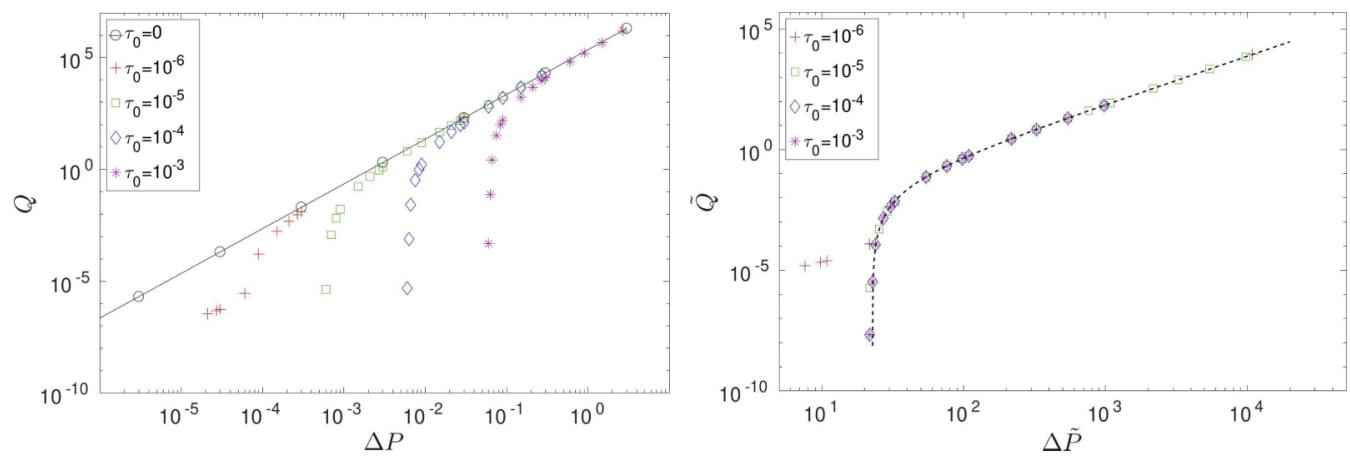

FIG. 11. Flow rate of the FCC packing as a function of the pressure difference for different values of $\tau_{0}$ 


\section{APPENDIX D: ENERGY MINIMIZATION}

In this section, we derive the minimization principle applied to Herschel-Bulkley fluid flow. From an arbitrary velocity field $\vec{u}$, we define the strain tensor operator

$$
\overline{\bar{\Delta}}_{i j}(\vec{u})=\frac{1}{2}\left(\partial_{i} u_{j}+\partial_{j} u_{i}\right)
$$

The stress tensor operator is then given by the Herschel-Bulkley law:

$$
\overline{\bar{\Sigma}}(\vec{u})=-P \overline{\bar{I}}+\overline{\bar{S}}(\vec{u}),
$$

with

$$
\overline{\bar{S}}(\vec{u})=\left[2 k \dot{\gamma}(\vec{u})^{n-1}+\frac{2 \tau_{0}}{\dot{\gamma}(\vec{u})}\right] \overline{\bar{\Delta}}(\vec{u})
$$

and $\dot{\gamma}(\vec{u})=\sqrt{2}\|\overline{\bar{\Delta}}(\vec{u})\|$.

We may now consider a rectangular porous medium of volume $V$, with length $L$ and base surface $S$ (inlet and outlet of the flow).

In the stationary regime, the bulk flow satisfies the momentum balance

$$
\forall i \in\{x, y, z\}, \sum_{j} \partial_{j} \overline{\bar{\Sigma}}_{i j}(\vec{u})+f_{i}=0
$$

where $\vec{f}$ is a body force.

We consider two possibilities for driving the flow: imposing a constant pressure difference $\Delta P$ or a constant flow rate $Q$.

\section{Constant pressure difference}

We first define the ensemble of admissible solutions $\Omega$ composed of every velocity field satisfying the divergence free $\sum_{i} \partial_{i} v_{i}=0$ and the no-slip condition at the solid interface $\vec{v}=\overrightarrow{0}$.

From Eq. (D4), we have for any velocity field of $\Omega$ :

$$
\begin{gathered}
\forall \vec{v} \in \Omega, \sum_{i} \int\left(\sum_{j} v_{i} \partial_{j} \overline{\bar{\Sigma}}_{i j}(\vec{u})+f_{i} v_{i}\right) d r^{3}=0, \\
\forall \vec{v} \in \Omega, \sum_{i} \oint \sum_{j} \overline{\bar{\Sigma}}_{i j}(\vec{u}) v_{j} n_{j} d S+\int\left(-\sum_{i j} \overline{\bar{\Sigma}}_{i j}(\vec{u}) \frac{1}{2}\left(\partial_{j} v_{i}+\partial_{i} v_{j}\right)+f_{i} v_{i}\right) d r^{3}=0,
\end{gathered}
$$

where the first term is a surface integral applied at the boundaries of normal $\vec{n}$. Thus,

$$
\forall \vec{v} \in \Omega, \sum_{i} \oint\left(t_{i} v_{i} d S\right)+\int f_{i} v_{i} d v=\int \overline{\bar{\Sigma}}_{i j}(\vec{u}) \overline{\bar{\Delta}}_{i j}(\vec{v}) d r^{3},
$$

where $t_{i}=\sum_{j} \overline{\bar{\Sigma}}_{i j} n_{j}$ is the imposed normal constraint applied to the fluid. The relation is usually called the principle of virtual work. Given an arbitrary virtual displacement, the virtual work of the internal constraints equals the virtual work of the external constraints.

We now define the internal energy function:

$$
\phi(\dot{\gamma}(\vec{v}))=\int_{0}^{\dot{\gamma}(v)}\left(k \dot{\gamma}(\vec{v})^{n}+\tau_{0}\right) d \dot{\gamma}=\frac{1}{n+1} k \dot{\gamma}^{n+1}(\vec{v})+\tau_{0} \dot{\gamma}(\vec{v}) .
$$


In the following, we will show that, for a given external constraint, the flow field $\vec{u}$, solution of Eq. (D4), is the minimum of the functional:

$$
\Phi^{P}[\vec{v}]=\int\{\phi[\dot{\gamma}(\vec{v})]-\vec{f} \cdot \vec{v}\} d v-\oint \vec{t} . \vec{v} d S .
$$

Indeed, for any $\vec{v} \in \Omega$, using the virtual work Eq. (D7), we have

$$
\Phi^{P}[\vec{v}]-\Phi^{P}[\vec{u}]=\int\{\phi[\dot{\gamma}(\vec{v})]-\phi[\dot{\gamma}(\vec{u})]-\overline{\bar{\Sigma}}(u):[\overline{\bar{\Delta}}(v)-\overline{\bar{\Delta}}(u)]\} d r^{3} .
$$

Using the concavity of the function $\phi$ and the free divergence, we deduce

$$
\begin{aligned}
\Phi^{P}[\vec{v}]-\Phi^{P}[\vec{u}] \geqslant & \int\left\{\phi^{\prime}[\dot{\gamma}(\vec{u})][\dot{\gamma}(\vec{v})-\dot{\gamma}(\vec{u})]+\left[k \dot{\gamma}(\vec{u})^{n}+\tau_{0}\right] \dot{\gamma}(\vec{u})\right. \\
& \left.-\left[2 k \dot{\gamma}(\vec{u})^{n-1}+\frac{2 \tau_{0}}{\dot{\gamma}(\vec{u})}\right] \overline{\bar{\Delta}}_{i j}(\vec{u}): \overline{\bar{\Delta}}_{i j}(\vec{v})\right\} d r^{3} .
\end{aligned}
$$

From the Cauchy-Schwarz inequality, $\overline{\bar{\Delta}}_{i j}(\vec{u}): \overline{\bar{\Delta}}_{i j}(\vec{v}) \leqslant\|\overline{\bar{\Delta}}(\vec{u})\|\|\overline{\bar{\Delta}}(\vec{v})\|$, we obtain

$$
\begin{aligned}
\forall \vec{v} \in \Omega, \Phi^{P}[\vec{v}]-\Phi^{P}[\vec{u}] & \geqslant \int\left\{\phi^{\prime}[\dot{\gamma}(\vec{u})] \dot{\gamma}(\vec{v})-\left[2 k \dot{\gamma}(\vec{u})^{n-1}+\frac{2 \tau_{0}}{\dot{\gamma}(\vec{u})}\right]\|\overline{\bar{\Delta}}(\vec{u})\|\|\overline{\bar{\Delta}}(\vec{v})\|\right\} d r^{3} \\
& \geqslant 0 .
\end{aligned}
$$

Thus, $\vec{u}$ is the global minimum of $\Phi^{P}[\vec{v}]$. This result is generic to any kind of external constraint or applied body force. For the particular case of the imposed pressure condition $[\vec{f}=0$ and $\oint \vec{t} \cdot \vec{v} d S=$ $\left.\left(P_{\text {in }}-P_{\text {out }}\right) Q(\vec{v})\right]$, the functional reads

$$
\Phi^{P}[\vec{v}]=\int\left[\frac{1}{n+1} k \dot{\gamma}^{n+1}(\vec{v})+\tau_{0} \dot{\gamma}(\vec{v})\right] d r^{3}-\left(P_{\text {in }}-P_{\text {out }}\right) Q(\vec{v}) .
$$

Introducing the nondimensional shear rate distribution $g_{u}(\vec{r}, \Delta P)=\frac{S \lambda}{Q(\vec{u})} \dot{\gamma}(\vec{u})$, it follows

$$
\begin{aligned}
\Phi[\vec{v}, \Delta P] & =\frac{1}{n+1} k \frac{Q^{n+1}(\vec{v}, \Delta P)}{S^{n+1} \lambda^{n+1}} \int g_{v}^{n+1}(\vec{r}, \Delta P) d r^{3} \\
& +\frac{\tau_{0}}{S \lambda} Q(\vec{v}, \Delta P) \int g_{v}(\vec{r}, \Delta P) d r^{3}-\Delta P Q(\vec{v}, \Delta P) .
\end{aligned}
$$

\section{Constant flow rate}

The idea is similar as in the previous case, however, it is necessary to define a more restrictive subspace of the admissible velocity field. We define $\Omega(Q)$ as the ensemble of velocity field satisfying

$$
\begin{aligned}
& \sum_{i} \partial_{i} v_{i}=0, \\
& v_{i}=0, \text { at the solid interfaces, } \\
& \sum_{j} \overline{\bar{\Sigma}}_{i j}(\vec{v}) n_{j}=-\lambda_{\text {in }}^{v} n_{i}, \text { across the inlet surface } S_{\text {in }}, \\
& \sum_{j} \overline{\bar{\Sigma}}_{i j}(\vec{v}) n_{j}=-\lambda_{\text {out }}^{v} n_{i}, \text { across the outlet surface } S_{\text {out }}, \\
& \sum_{i} \oint_{S_{\text {out }}} v_{i} n_{i} d S=-\sum_{i} \oint_{S_{\text {in }}} v_{i} n_{i} d S=Q
\end{aligned}
$$

for any real number $\left(\lambda_{\text {in }}^{v}, \lambda_{\text {out }}^{v}\right)$. 
Multiplying Eq. (D4) by any $\vec{v} \in \Omega(Q)$, we obtain (assuming $\vec{f}=\overrightarrow{0}$ )

$$
\left.\forall \vec{v} \in \Omega(Q), \sum_{i} \oint \sum_{j} \overline{\bar{\Sigma}}_{i j}(\vec{u}) v_{j} n_{j} d S-\int \sum_{i j} \overline{\bar{\Sigma}}_{i j}(\vec{u}) \overline{\bar{\Delta}}_{i j}(\vec{v})\right) d r^{3}=0 .
$$

Thus,

$$
\forall \vec{v} \in \Omega(Q),\left(\lambda_{\text {in }}^{u}-\lambda_{\text {out }}^{u}\right) Q-\int \sum_{i j} \overline{\bar{\Sigma}}_{i j}(\vec{u}) \overline{\bar{\Delta}}_{i j}(\vec{v}) d r^{3}=0 .
$$

Substracting this relation applied to $\vec{u}$ leads then to

$$
\forall \vec{v} \in \Omega(Q), \int \sum_{i j} \overline{\bar{\Sigma}}_{i j}(\vec{u}) \overline{\bar{\Delta}}_{i j}(\vec{v}-\vec{u}) d r^{3}=0,
$$

or

$$
\forall \vec{v} \in \Omega(Q), \int \sum_{i j} \overline{\bar{S}}_{i j}(\vec{u}) \overline{\bar{\Delta}}_{i j}(\vec{v}-\vec{u}) d r^{3}=0,
$$

because of the free divergence of $\vec{u}$ and $\vec{v}$.

We will now demonstrate that $\vec{u}$ is the minimum of the functional:

$$
\Phi^{Q}[\vec{v}]=\int \phi[\dot{\gamma}(\vec{v})] d r^{3}=\int\left[\frac{1}{n+1} k \dot{\gamma}^{n+1}(\vec{v})+\tau_{0} \dot{\gamma}(\vec{v})\right] d r^{3} .
$$

Indeed, we have

$$
\forall \vec{v} \in \Omega(Q), \Phi^{Q}[\vec{v}]-\Phi^{Q}[\vec{u}] \geqslant \int\left\{\phi^{\prime}[\dot{\gamma}(\vec{u})][\dot{\gamma}(\vec{v})-\dot{\gamma}(\vec{u})]\right\} d r^{3},
$$

from the concavity of $\phi[\gamma(\vec{v})]$. And thus

$$
\begin{aligned}
\forall \vec{v} \in \Omega(Q), \Phi^{Q}[\vec{v}]-\Phi^{Q}[\vec{u}] & \geqslant \int\left\{\left[k \dot{\gamma}(\vec{u})^{n-1}+\frac{\tau_{0}}{\dot{\gamma}(\vec{u})}\right]\left[\dot{\gamma}(\vec{v}) \dot{\gamma}(\vec{u})-\dot{\gamma}(\vec{u})^{2}\right]\right\} d r^{3} \\
& \geqslant \int\left\{\left[k \dot{\gamma}(\vec{u})^{n-1}+\frac{\tau_{0}}{\dot{\gamma}(\vec{u})}\right]\left[2\|\overline{\bar{\Delta}}(\vec{v})\|\|\overline{\bar{\Delta}}(\vec{u})\|-2\|\overline{\bar{\Delta}}(\vec{u})\|^{2}\right]\right\} d r^{3} \\
& \geqslant \int\left\{2\left[k \dot{\gamma}(\vec{u})^{n-1}+\frac{\tau_{0}}{\dot{\gamma}(\vec{u})}\right] \sum_{i j}\left[\overline{\bar{\Delta}}_{i j}(\vec{v})-\overline{\bar{\Delta}}_{i j}(\vec{u})\right] \overline{\bar{\Delta}}_{i j}(\vec{u})\right\} d r^{3} \\
& \geqslant \int \sum_{i j} S_{i j}(\vec{u}) \overline{\bar{\Delta}}_{i j}(\vec{v}-\vec{u}) d r^{3} \\
& \geqslant 0,
\end{aligned}
$$

where we have applied the Cauchy-Schwartz inequality and the relation Eq. (D16).

[1] L. Talon and D. Bauer, On the determination of a generalized Darcy equation for yield-stress fluid in porous media using a lattice-Boltzmann TRT scheme, Eur. Phys. J. E 36, 139 (2013).

[2] V. M. Entov, On some two-dimensional problems of the theory of filtration with a limiting gradient, Prikl. Mat. Mekh. 31, 820 (1967). 
[3] T. Al-Fariss and K. L. Pinder, Flow through porous media of a shear-thinning liquid with yield stress, Can. J. Chem. Eng. 65, 391 (1987).

[4] A. Ambari, M. Benhamou, S. Roux, and E. Guyon, Distribution des tailles de pores d'un milieu poreux déterminée par l'écoulement d'un fluide à seuil, Comptes rendus de l'Académie des sciences. Série 2, Mécanique, Physique, Chimie, Sciences de l'univers, Sciences de la Terre 311, 1291 (1990).

[5] G. G. Chase and P. Dachavijit, A correlation for yield stress fluid flow through packed beds, Rheol. Acta 44, 495 (2005).

[6] M. Chen, W. Rossen, and Y. C. Yortsos, The flow and displacement in porous media of fluids with yield stress, Chem. Eng. Sci. 60, 4183 (2005).

[7] G. G. Chase and P. Dachavijit, An experimental study of electrorheological fluid flow through a packed bed of glass beads, Transp. Porous Media 72, 25 (2008).

[8] T. Sochi and M. Blunt, Pore-scale network modeling of Ellis and Herschel-Bulkley fluids, J. Pet. Sci. Eng. 60, 105 (2008).

[9] M. Balhoff, D. Sanchez-Rivera, A. Kwok, Y. Mehmani, and M. Prodanović, Numerical algorithms for network modeling of yield stress and other non-newtonian fluids in porous media, Transp. Porous Media 93, 363 (2012).

[10] T. Chevalier, C. Chevalier, X. Clain, J. C. Dupla, J. Canou, S. Rodts, and P. Coussot, Darcy's law for yield stress fluid flowing through a porous medium, J. Non-Newtonian Fluid Mech. 195, 57 (2013).

[11] T. Chevalier, S. Rodts, X. Chateau, C. Chevalier, and P. Coussot, Breaking of non-Newtonian character in flows through a porous medium, Phys. Rev. E 89, 023002 (2014).

[12] A. R. de Castro, A. Omari, A. Ahmadi-Sénichault, and D. Bruneau, Toward a new method of porosimetry: Principles and experiments, Transp. Porous Media 101, 349 (2014).

[13] J. Bleyer and P. Coussot, Breakage of non-Newtonian character in flow through a porous medium: Evidence from numerical simulation, Phys. Rev. E 89, 063018 (2014).

[14] S. Nash and D. A. S. Rees, The effect of microstructure on models for the flow of a bingham fluid in porous media: one-dimensional flows, Transp. Porous Media 116, 1073 (2017).

[15] S. Shahsavari and G. McKinley, Mobility and pore-scale fluid dynamics of rate-dependent yield-stress fluids flowing through fibrous porous media, J. Non-Newtonian Fluid Mech. 235, 76 (2016).

[16] L. Talon, H. Auradou, and A. Hansen, Effective rheology of Bingham fluids in a rough channel, Front. Phys. 2, 24 (2014).

[17] T. Chevalier and L. Talon, Generalization of Darcy's law for Bingham fluids in porous media: From flow-field statistics to the flow-rate regimes, Phys. Rev. E 91, 023011 (2015).

[18] W. H. Herschel and R. Bulkley, Konsistenzmessungen von Gummi-Benzollösungen, Kolloid-Zeitschrift 34, 291 (1926).

[19] J. M. Piau, Carbopol gels: Elastoviscoplastic and slippery glasses made of individual swollen sponges: Meso-and macroscopic properties, constitutive equations and scaling laws, J. Non-Newtonian Fluid Mech. 144, 1 (2007).

[20] P. Coussot, Yield stress fluid flows: A review of experimental data, J. Non-Newtonian Fluid Mech. 211, 31 (2014).

[21] I. Ginzburg, A free-surface lattice Boltzmann method for modeling the filling of expanding cavities by Bingham fluids, Proc. R. Soc. London A 360, 453 (2002).

[22] I. Ginzburg, F. Verhaeghe, and D. d'Humieres, Two-relaxation-time lattice Boltzmann scheme: About parametrization, velocity, pressure, and mixed boundary conditions, Commun. Comput. Phys. 3, 427 (2008).

[23] I. Ginzburg, F. Verhaeghe, and D. d'Humieres, Study of simple hydrodynamic solutions with the tworelaxation-times lattice Boltzmann scheme, Commun. Comput. Phys. 3, 519 (2008).

[24] T. Papanastasiou, Flows of materials with yield, J. Rheol. (1978-present) 31, 385 (1987).

[25] H. B. Ly, V. Monchiet, and D. Grande, Computation of permeability with fast fourier transform from 3D digital images of porous microstructures, Int. J. Numer. Methods Heat Fluid Flow 26, 1328 (2016).

[26] G. Duvaut and J. L. Lions, Inequalities in Mechanics and Physics (Springer, Berlin, 1976).

[27] J. B. Keller, L. Rubenfeld, and J. Molyneux, Extremum principles for slow viscous flows with applications to suspensions, J. Fluid Mech. 30, 97 (1967). 
[28] A. Roustaei and I. A. Frigaard, The occurrence of fouling layers in the flow of a yield stress fluid along a wavy-walled channel, J. Non-Newtonian Fluid Mech. 198, 109 (2013).

[29] A. Roustaei, T. Chevalier, L. Talon, and I. A. Frigaard, Non-Darcy effects in fracture flows of a yield stress fluid, J. Fluid Mech. 805, 222 (2016).

[30] L. Talon, D. Bauer, N. Gland, S. Youssef, H. Auradou, and I. Ginzburg, Assessment of the two relaxation time lattice-Boltzmann scheme to simulate Stokes flow in porous media, Water Resour. Res. 48, W04526 (2012). 